\title{
A Density Functional Theory Study for the Adsorption of Various Gases on a Caesium- Exchanged Trapdoor Chabazite
}

\author{
Jin Shang ${ }^{\mathrm{a}, \mathrm{b}_{*}}$, Gang $\mathrm{Li}^{\mathrm{c}}$, Paul A. Webley ${ }^{\mathrm{a}, \mathrm{b} *}$, and Jefferson Z. Liu ${ }^{\mathrm{d} *}$ \\ ${ }^{a}$ Cooperative Research Centre for Greenhouse Gas Technologies \\ ${ }^{\mathrm{b}}$ Department of Chemical and Biomolecular Engineering, The University of Melbourne, \\ Victoria 3010, Australia \\ 'School of Mechanical and Chemical Engineering, The University of Western Australia, \\ Crawley, WA 6009, Australia \\ ${ }^{\mathrm{d} D e p a r t m e n t}$ of Mechanical and Aerospace Engineering, Monash University, Clayton, \\ Victoria 3800, Australia \\ Corresponding authors: jin.shang@ @nimelb.edu.au, Tel: +61 3 83446640; \\ paul.webley@unimelb.edu.au, Tel: +61 3 90357873; zhe.liu@ monash.edu, Tel: +61 3990 \\ 53627
}

\begin{abstract}
Rational design and development of porous materials for adsorptive gas separation gains ever-increasing attention as industrial applications, such as carbon capture and natural gas purification, always require more energy-efficient processes with adsorbents providing high selectivity. Zeolite molecular sieves represent a class of such desirable adsorbents. Our recently discovered molecular trapdoor mechanism in zeolites allows for unprecedented high selectivity and affords designability for versatile adsorbents. In this work, we presented a route for identifying the molecular trapdoor mechanism and predicting the gas separation feasibility using density functional theory calculations, based on a typical molecule trapdoor zeolite - caesium-exchanged chabazite with silicon to aluminium ratio of 3 . We established criteria to assess the viability for "door-open" process by examining the dependence of energy barriers for the movement of "door-keeping" cation in the presence of different gases. Calculations at the standard PBE level and the van der Waals DFT levels were carried out. This theoretical route could serve as a standard method to study and develop other molecular trapdoor zeolites.
\end{abstract}

Keywords: chabazite zeolite $\cdot$ adsorption $\cdot$ molecular trapdoor $\cdot$ DFT 


\section{Introduction}

Gas separations using microporous materials represents an ever-booming field, especially in recent years, as carbon capture attracts increasing attention due to the pressing concern on energy crisis and environmental protection [1, 2]. For carbon capture, such an energyefficient process requires high-performance adsorbents to selectively remove $\mathrm{CO}_{2}$ from other molecules such as $\mathrm{N}_{2}, \mathrm{CH}_{4}, \mathrm{H}_{2}$, and $\mathrm{O}_{2}$. Zeolites serve as a primary class of candidate adsorbents for large-scale gas separations owning to multiple advantages such as high selectivity, low cost, as well as high thermal and mechanical stability [3]. These highly porous aluminosilicates possess three-dimensional structure of molecular dimension, thus being known as "molecular sieves". They could yield extremely high selectivity attributed to the molecular sieving effect: the host admits small guests in but excludes large ones out through its pore aperture [4].

However, this conventional explanation for the molecular sieving mechanism deserves a closer scrutiny when accounting for a host aperture coordinated by extraframework cations. In this case, a molecular trapdoor mechanism provides a more reasonable picture: a cationkept zeolite aperture only exclusively admits certain molecules regardless of size [5-7]. We demonstrated this mechanism by investigating $\mathrm{CO}_{2}, \mathrm{~N}_{2}$, and $\mathrm{CH}_{4}$ separation on chabazite zeolites. Chabazite $[8,9]$ features eight-membered ring $(8 \mathrm{MR})$ apertures that act as its only access for guest molecules; but in specifically tailored chabazites the guests are blocked by extraframework cations such as $\mathrm{K}^{+}$and $\mathrm{Cs}^{+}$, which coordinate energetically favourably in the center of the $8 \mathrm{MRs}$. For a guest molecule to enter the supercavity, the door-keeping cation has to move away from the center of the $8 \mathrm{MR}$ aperture. Our previous studies showed that $\mathrm{CO}_{2}$ has "stronger" interaction strength with the door-keeping cations than $\mathrm{N}_{2}$ and $\mathrm{CH}_{4}$ molecules and thus can significantly flatten the potential well of the cation. At a certain temperature (corresponding to a certain kinetic energy value for the cation), the thermal excitation would lead to the temporary and reversible deviation of the cations away from the center of the $8 \mathrm{MR}$, giving way to $\mathrm{CO}_{2}$ molecules to hop into the adjacent cages.

This novel molecular trapdoor mechanism opens up a new avenue for designing microporous materials with steered selectivity. However, a rational design requires detailed understanding at the atomic level, aside from trial and error procedures in the lab. Computer simulations provide indispensable tools to investigate and predict the physical chemistry properties at the 
atomic level. In this work, we employed density functional theory (DFT) calculations to study the molecular trapdoor mechanism occurring to a Cs-exchanged chabazite. Three typical small size gas molecules, $\mathrm{CO}_{2}, \mathrm{~N}_{2}$, and $\mathrm{CH}_{4}$ which are relevant to carbon capture applications, were adopted in our theoretical study. We established criteria to assess the viability of "door-open" process. In the end of this manuscript, the criteria were used to predict the selective adsorption of several other small gas molecules in the trapdoor chabazite. This work could afford a standard route for the theoretical design of the molecular trapdoor materials.

\section{Methods}

\subsection{Structure Model Setup}

Chabazite [8] consists of double-six ring (D6R) units arranged in layers that are linked together by tilted 4-membered rings (4MRs). The three-dimensional pore structure comprises large ellipsoidal supercavities of $6.7 \times 10 \AA$, each of which is accessed by six eightmembered rings $(8 \mathrm{MRs})$ of $3.8 \times 3.8 \AA$ (Fig. 1). Previous studies [10-12] have shown that four types of general extraframework cation sites exist in dehydrated chabazite: one at the centre of the D6R prism (SI), one at the triad axis of the D6R prism but displaced to the supercavity (SII), one in the supercavity at the corner of the 4MR window of the D6R (SIII), and one at the center of the 8MR window (SIII').

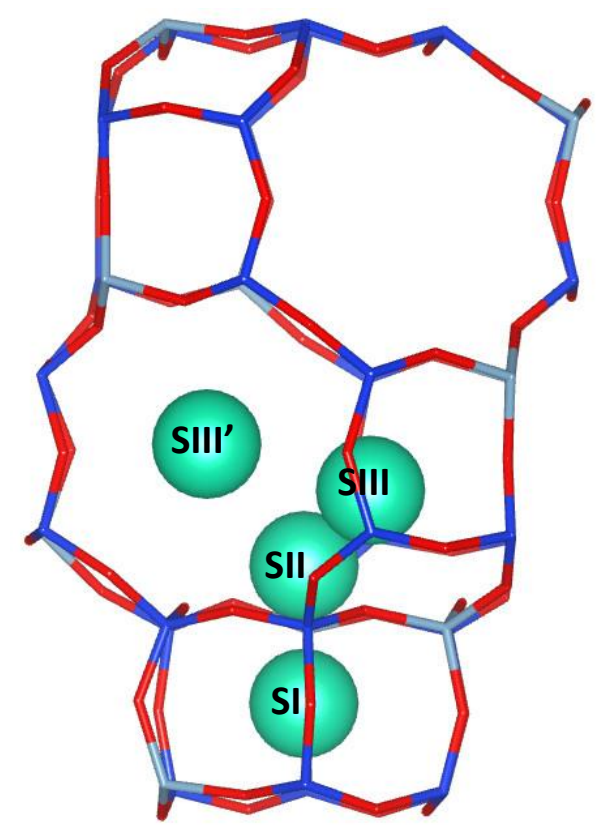


Fig. 1. Schematic representation of chabazite crystal structure and the extraframework cation sites. Double-six ring prisms (D6Rs) connected by tilted four-membered rings (4MRs) form a three-dimensional structure, generating eight-membered rings (8MRs) as the only access to the crystal interior. Four sites exist for extraframework cations: inside the center of a D6R (SI); inside the supercavity above a D6R (SII); inside the supercavity next to a 4MR (SIII); in the center of an 8MR (SIII'). Color scheme: Red, O; Blue: Si; Grey, Al; Aqua, Cs.

Pure-silica chabazite was adopted as the starting point where the structural parameters for a conventional unit cell (referred to as unit cell hereafter), which consists of three double sixring prisms or one and a half chabazite supercages, were taken from an experimental work [13]. Specifically, $a=13.92779 \AA, b=13.94511 \AA, c=15.12131 \AA, \alpha=90.0929^{\circ}, \beta=$ $89.9757^{\circ}, \gamma=120.3650^{\circ}$. To introduce extraframework cations such as $\mathrm{K}^{+}$and $\mathrm{Cs}^{+}$into chabazite, a certain amount of silicon atoms were replaced by aluminium atoms to generate negatively charged chabazite frameworks with $\mathrm{Si} / \mathrm{Al}$ ratio of 35:1 (r35CHA) and 3:1 (r3CHA), respectively. The unit cell formula is $\mathrm{K}_{1}\left[\mathrm{Al}_{1} \mathrm{Si}_{35} \mathrm{O}_{72}\right]$ ) for $\mathbf{r 3 5 K C H A}$, $\mathrm{Cs}_{1}\left[\mathrm{Al}_{1} \mathrm{Si}_{35} \mathrm{O}_{72}\right]$ ) for $\mathbf{r 3 5 C s C H A}$, and $\mathrm{Cs}_{9}\left[\mathrm{Al}_{9} \mathrm{Si}_{27} \mathrm{O}_{72}\right]$ for $\mathbf{r} 3 \mathrm{CsCHA}$. In our study, the r35CHA was used to determine cation site preference, which could be directly compared with literature results. The r3CHA represents our experimentally investigated chabazite counterpart, in which the trapdoor mechanism is enabled by considering the cations at relevant sites (SIII') [5].

Given that the $\mathrm{Al}$ atoms introduce negative charges into chabazite frameworks, the extraframework cations were initially placed at the cation sites in the vicinity of $\mathrm{Al}$ atoms in our DFT calculations. For either r35KCHA or r35CsCHA, since there is only one Al per unit cell, we placed this $\mathrm{Al}$ at an intersection of an $8 \mathrm{MR}$, a $6 \mathrm{MR}$, and a $4 \mathrm{MR}$. This allows us to generate a structure with a $\mathrm{K}^{+}$or $\mathrm{Cs}^{+}$cation placed at each of the possible cation sites (see Figure 1 for details). By comparing the total energy results $\left(E_{\text {total }}\right)$ of the obtained different crystal structures, i.e., the total energy with the cation at site SI $\left(E_{\text {total }}(\mathrm{SI})\right)$, the total energy with the cation at site SII $\left(E_{\text {total }}(\mathrm{SII})\right), E_{\text {total }}(\mathrm{SIII})$, and the total energy with the cation at site SIII' $\left(E_{\text {total }}(\right.$ SIII')), we could determine the site preference as per the rule, i.e., a higher total energy value indicates an unfavourable cation site. In the case of $\mathbf{r} 3 \mathrm{CsCHA}$, there are many different ways to place the $9 \mathrm{Al}$ atoms in one unit cell. In order to calculate the energy barrier 
for $\mathrm{Cs}^{+}$cation migrating from site SIII' to site SII with different number of $\mathrm{Al}$ atoms in the corresponding 8MRs and 6MRs, we considered all the possible Al distributions (Figure 5).

For the gas-chabazite complex calculations, the structures were generated by placing a certain loading of gas molecules next to the target cations in supercavities of chabazite crystals. The gas molecule positions were selected so that both cation-gas and gas-gas distances were approximately $3.0 \AA$.

\subsection{Computational Setup}

$A b$ initio density functional theory (DFT) calculations were employed to determine the chabazite structure, cation location and occupancy, as well as gas adsorption configuration. We used the Vienna Ab initio Simulation Package (VASP) [15] with the generalized gradient approximation (GGA) [16] and the projector augmented waves (PAW) approach [17]. The cut-off energy of the plane wave basis-set was $600 \mathrm{eV}$. A gamma point only k-point mesh was used for one unit cell of chabazite (including three double-six ring prisms or one and a half chabazite supercage). Such cut-off energy and k-point mesh have been tested to ensure the total energy convergence within $1 \mathrm{meV} /$ atom. The atomic positions were optimized with the conjugate gradient method until the forces acting on atoms were below $0.015 \mathrm{eV} / \AA$, as suggested by Göltl and Hafner [14]. We applied the nudged-elastic-band (NEB) method [22] for energy barrier calculations.

For the bare chabazite systems, the crystal structure were fully relaxed at PBE level since the dominant interaction in chabazite is the covalent bonding between silicon/aluminium and oxygen atoms and van der Waals dispersion interactions do not play an important role [14]. To more accurately account for the van der Waals interaction in the gas-chabazite complexes, dispersion-corrected DFT calculations with the optB86b-vdW functional scheme [18-20] was adopted because this scheme was determined to be the most appropriate one in our gaschabazite system in a previous study [21]. Note that in our optB86b-vdW functional calculations, the gas-chabazite complexes fully relaxed at the PBE level were taken as the starting point.

\section{Results and Discussion}




\subsection{Validation of Chabazite Model}

We tested our chabazite model system by comparing the $\mathrm{K}^{+}$cation site preference in r35KCHA with those reported in literatures. This $\mathrm{Si} / \mathrm{Al}$ ratio corresponds to replacing only one $\mathrm{Si}$ atom by one $\mathrm{Al}$ atom in one unit cell, which can be taken as a dilute exchanging case. Figure 2 shows the relative total energy of the $\mathrm{r35KCHA}$ with the $\mathrm{K}^{+}$cation located at different sites. Clearly, the $\mathrm{K}^{+}$cation favours to reside at these sites in a sequence SIII' $>$SII $>$ SIII > SI, which agrees with a previous simulation study [23]. We can rationalize the site preference by considering the possible coordination numbers between $\mathrm{K}^{+}$cation and framework $\mathrm{O}$ atoms. A higher coordination number often leads to a more stable configuration [24]. As shown in Fig. S1: $\mathrm{K}^{+}$could coordinate with eight $\mathrm{O}$ atoms at site SIII' (Fig. S1a), six at site SII (Fig. S1b), four at site SIII (Fig. S1c), and twelve at site I (Fig. S1d). Note that the highest coordination number at site SI does not lead to the most stable configuration rather the least stable because the volume enclosed in the D6R is too small for the $\mathrm{K}^{+}$cation.

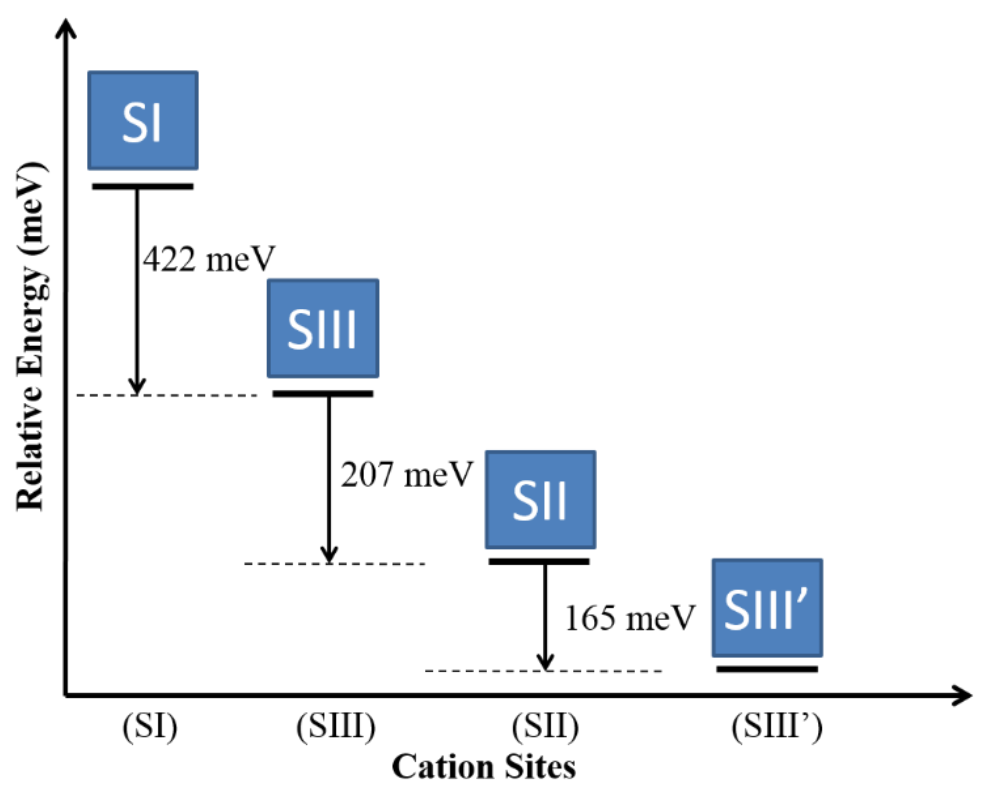

Fig. 2. Relative total energy of $\mathbf{r} 35 \mathrm{KCHA}$ crystals with $\mathrm{K}^{+}$cation located at different sites. Cleary the site SIII' is the most stable cation site for $\mathrm{K}^{+}$.

\subsection{Determination of $\mathrm{Cs}^{+}$Cation Site Preference}

On the basis of the verified chabazite model - r35KCHA, we built r35CsCHA by simply replacing $\mathrm{K}^{+}$with $\mathrm{Cs}^{+}$and investigated the $\mathrm{Cs}^{+}$cation site preference. Note that site I was not 


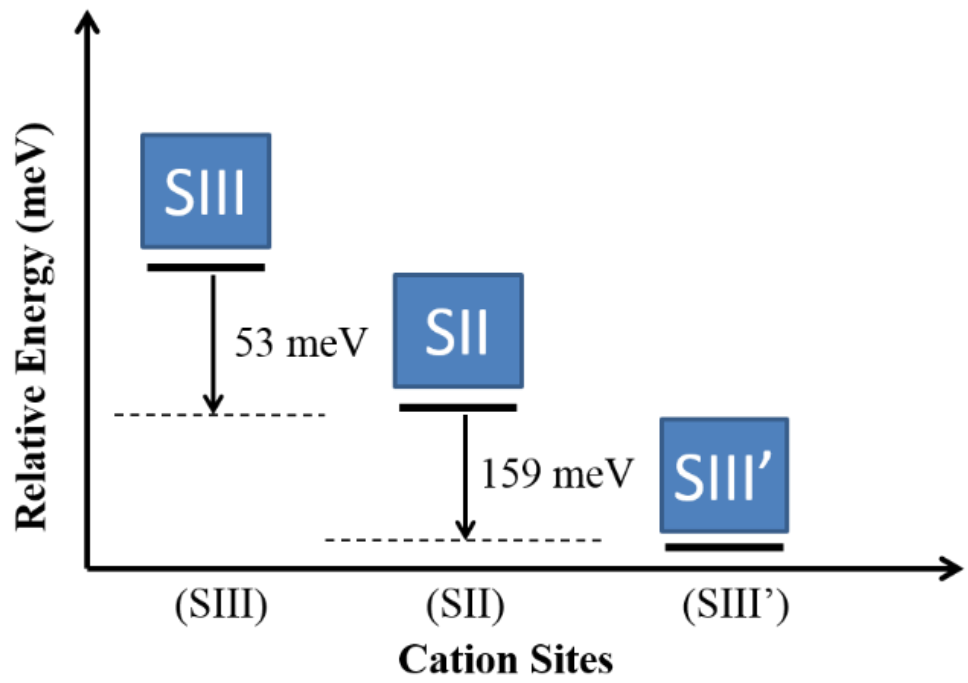

Fig. 3. Relative total energy of $\mathbf{r 3 5 C s C H A}$ crystals with $\mathrm{Cs}^{+}$cation located at different sites. Site SIII' is the most stable cation site for $\mathrm{Cs}^{+}$.

In order to examine if the site preference in the $\mathbf{r 3 5 C s C H A}$ applies to that in the $\mathbf{r} 3 \mathrm{CsCHA}$, we carried out two sets of calculations. The first set includes three different configurations. The first configuration has all nine $\mathrm{Cs}^{+}$cations located at the nine SIII' sites. In the second and third configurations, one $\mathrm{Cs}^{+}$cation is moved from SIII' to SII and SIII, respectively. Table 1 compares the total energy results, showing an occupation sequence, i.e., SIII' > SII > SIII, similar to that in $\mathbf{r 3 5 C s C H A}$, despite the increased cation density. This agreement suggests that the coexistence of multiple $\mathrm{Cs}^{+}$cations in one unit cell does not affect the site preference of a single $\mathrm{Cs}^{+}$cation. 
In our second set of calculations, we moved one, two, and three $\mathrm{Cs}^{+}$cations from SIII' to site II, respectively. Table 2 lists the total energy results. Clearly all nine $\mathrm{Cs}^{+}$cations prefer to sit at site III'. This cation distribution pattern agrees well with our recent experimental results on Cs-exchanged chabazite with a $\mathrm{Si} / \mathrm{Al}$ ratio of $\sim 2.5$ as determined by synchrotron powder XRD [5]. A previous experimental study showed that $\mathrm{Cs}^{+}$cations preferentially occupy all the SIII' sites in a dehydrated Cs form chabazite with similar Si/Al ratio of 2.5 [24]. Another experimental study also showed that large cation, $\mathrm{K}^{+}$therein, favours site SIII' in a chabazite with $\mathrm{Si} / \mathrm{Al}$ ratio of $\sim 2.5$ [25], which is also consistent with our results in $\mathbf{r} 3 \mathrm{CsCHA}$. The excellent agreement with experimental results justifies the reliability of our structural models and DFT computational approach, allowing us to carry out further computational studies to reveal material properties and in-depth fundamental gas admission mechanism that cannot be captured by the use of experimental methods.

$\mathrm{Cs}^{+}$cation stably coordinates at site SIII' and is unlikely to hop to other sites under ambient temperature. Our previous in situ synchrotron PXRD experimental study clearly showed that $\mathrm{Cs}^{+}$cation stably coordinates at the pore aperture site (site SIII') with an $100 \%$ site occupancy up to $303 \mathrm{~K}$ [5]. Interestingly, the immobile behaviour of $\mathrm{Cs}^{+}$cation is different from the mobile $\mathrm{Na}^{+}$cation in MOR zeolite [26]. This could be because $\mathrm{Cs}^{+}$cation is heavier and larger than $\mathrm{Na}^{+}$cation.

Table 1. DFT total energy results for the r3CsCHA unit cell with eight $\mathrm{Cs}^{+}$cations located at SIII' and the ninth $\mathrm{Cs}^{+}$cation located at SIII', SII, and SIII, respectively.

\begin{tabular}{llll}
\hline Cs distribution & $9 \mathrm{Cs}^{+}(\mathrm{SIII})$ & $8 \mathrm{Cs}^{+}(\mathrm{SIII})$ & $8 \mathrm{Cs}^{+}(\mathrm{SIII})$ \\
& & $1 \mathrm{Cs}^{+}(\mathrm{SII})$ & $1 \mathrm{Cs}^{+}(\mathrm{SIII})$ \\
\hline Total Energy $(\mathrm{eV})$ & -872.696 & -872.188 & -871.578 \\
\hline
\end{tabular}

Table 2. DFT total energy results for the $\mathbf{r} 3 \mathrm{CsCHA}$ unit cell with $\mathrm{Cs}^{+}$cations distributed at SIII' and SII sites.

\begin{tabular}{lllll}
\hline \multirow{2}{*}{ Cs distribution } & $9 \mathrm{Cs}^{+}\left(\mathrm{SIII}^{\prime}\right)$ & $\begin{array}{c}8 \mathrm{Cs}^{+}(\mathrm{SIII}) \\
1 \mathrm{Cs}^{+}(\mathrm{SII})\end{array}$ & $\begin{array}{l}7 \mathrm{Cs}^{+}(\mathrm{SIII}) \\
2 \mathrm{Cs}^{+}(\mathrm{SII})\end{array}$ & $\begin{array}{l}6 \mathrm{Cs}^{+}(\mathrm{SIII}) \\
3 \mathrm{Cs}^{+}(\mathrm{SII})\end{array}$ \\
\hline Total Energy $(\mathrm{eV})$ & -872.696 & -872.188 & -871.792 & -871.152 \\
\hline
\end{tabular}




\subsection{Determination of $\mathrm{Cs}^{+}$Cation Movement Path}

As the relative stability of different cation sites is clear, we can propose possible path for cation movement upon gas adsorption. Note that the most stable site SIII' directly relates to gas admission through a chabazite $8 \mathrm{MR}$ aperture. In our previous work we proposed that for gas molecules such as $\mathrm{CO}_{2}, \mathrm{~N}_{2}$, and $\mathrm{CH}_{4}$ to pass through an $8 \mathrm{MR}$ pore aperture, the doorkeeping cation (e.g., $\mathrm{Cs}^{+}$residing at SIII') has to move away from this site [5]. Given site SII represents the second most stable site for $\mathrm{Cs}^{+}$cation following site SIII', we treated the path from SIII' to SII as cation movement path. No matter if the cation permanently moves from SIII' to SII (migration) or temporarily and reversibly moves away from SIII' toward SII (deviation), we can use the energy barrier associated with the cation movement path to assess the difficulty for this process to occur.

We started from the relatively simple case $-\mathrm{Cs}^{+}$movement in $\mathbf{r 3 5 C s C H A}$. Using NEB method, we calculated the energy barrier $\Delta E_{\mathrm{b}}$ for one $\mathrm{Cs}^{+}$cation moving from site SIII' to site SII (shown in Fig. 4a). Clearly, the $\mathrm{Cs}^{+}$cation has to climb a "hill" between site SIII" and site SII in order to reach this second most stable site (Fig. 4b).

(a)

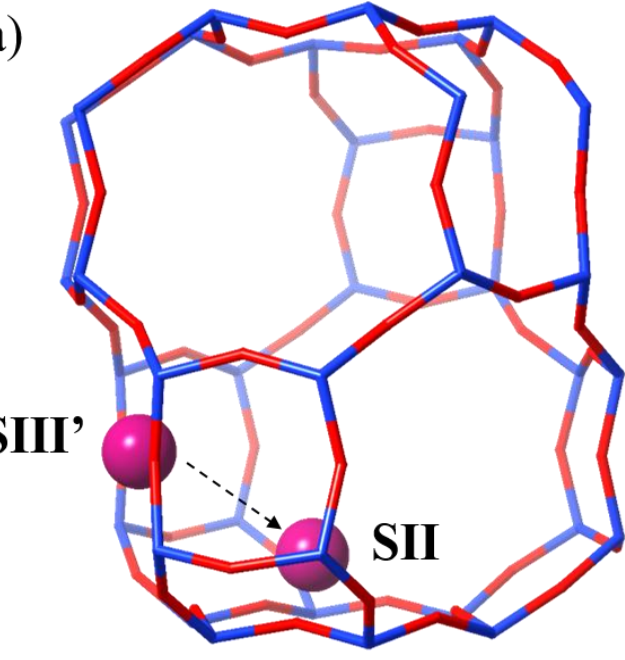

(b)

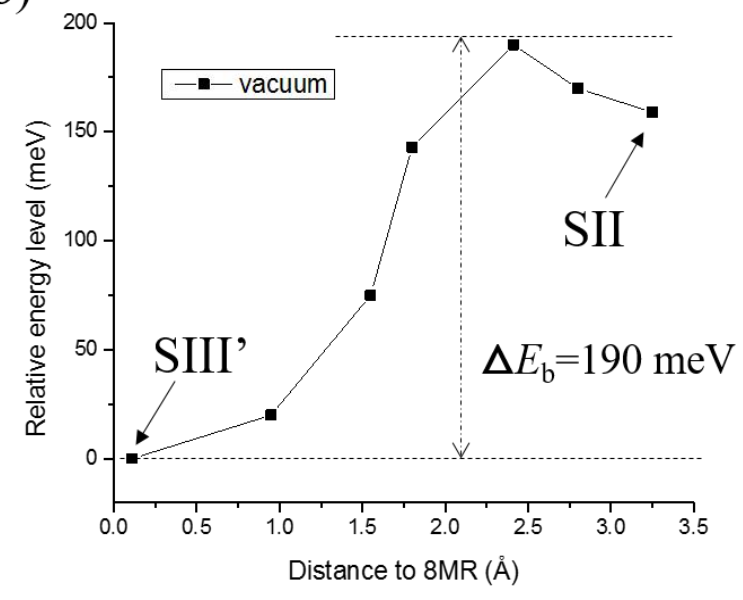

Fig. 4. (a) Sketch to illustrate the migration path of $\mathrm{Cs}^{+}$from SIII' to SII in case of r35CsCHA. (b) Relative total energy results at different positions in the migration path calculated by using the NEB method. 
Determining the $\Delta E_{\mathrm{b}}$ for $\mathrm{Cs}^{+}$migration in $\mathbf{r} 3 \mathrm{CsCHA}$ would be more complicated because various Al distribution possibilities should affect the energy barrier results. Due to uneven $\mathrm{Si} / \mathrm{Al}$ distribution, r3CsCHA has different 8MRs and 6MRs in terms of different number of $\mathrm{Al}$ atoms contained in a given ring, leading to different stability for $\mathrm{Cs}^{+}$cation at different sites SIII' and SII, respectively. For example, there exist 8MRs containing one, two, and three $\mathrm{Al}$ atoms, respectively; and 6MRs containing one and two $\mathrm{Al}$ atoms, respectively. The combination of various pairs of $8 \mathrm{MR}$ and $6 \mathrm{MR}$, in principle, should lead to different energy barrier results. Among them, the lowest one is the most important case since the 8MRs would be opened (resulting from the cation migration) at a lower temperature to admit gas molecules. Unfortunately, it is very time consuming to do DFT NEB calculations for all cases to identify the one with the lowest energy barrier. A time efficient semi-quantitative method is desirable.

We note that in the case of $\mathbf{r 3 5 C S C H A}$, the energy barrier $\left(\Delta E_{\mathrm{b}}\right)$ of $190 \mathrm{meV}$ is comparable to the energy difference $(\Delta E)$ between sites SIII' and SII, namely, $159 \mathrm{meV}$ (Fig. 3). In addition, the cation migration path is in the vicinity of the $8 \mathrm{MR}$ and $6 \mathrm{MR}$. We postulate that the energy barrier and energy difference should be largely determined by the structure of these two rings (i.e., different number of $\mathrm{Al}$ atoms). There should be a correlation between $\Delta E_{\mathrm{b}}$ and $\Delta E$. Therefore, here we proposed to use $\Delta E$ as a semi-quantitative measure for the energy barrier $\Delta E_{\mathrm{b}}$ to characterize the difficulty in cation movement (from SIII' towards SII). Figure 5 shows the energy difference in all six types of pair combinations of $8 \mathrm{MR}$ and $6 \mathrm{MR}$. $\mathrm{A} \mathrm{Cs}^{+}$ cation migration from a $3-\mathrm{Al}$-contained $8 \mathrm{MR}$ to a 1 -Al-contained $6 \mathrm{MR}$ yields the highest energy difference (938 meV). The cation migration from a 2-Al-contained 8MR to a 2-Alcontained $6 \mathrm{MR}(501 \mathrm{meV})$ or from a 1-Al-contained 8MR to a 2-Al-contained 6MR (508 $\mathrm{meV}$ ) has a quite close small $\Delta E$ value. Since cation energetically favours to coordinate to a ring with more charges via substitution of $\mathrm{Si}$ atom by $\mathrm{Al}$ atom, a cation migration from an $\mathrm{Al}-$ rich $8 \mathrm{MR}$ to an Al-lean $6 \mathrm{MR}$ should experience the largest $\Delta E$ and vice versa. This is in a general consistency with our DFT results.

We carried out NEB calculation for $\Delta E_{\mathrm{b}}$ for $\mathrm{Cs}^{+}$cation migration under vacuum for 1-Alcontained $8 \mathrm{MR}$ to a 2-Al-contained 6MR. The resulting energy barrier $\left(\Delta E_{\mathrm{b}}=651 \mathrm{meV}\right)$ (Fig. 7) is comparable to the corresponding energy difference ( $\Delta E=508 \mathrm{meV}$ ) (Fig. 5), indicating 
that $\Delta E$ can be adopted as a convenient semi-quantitative measure to efficiently determine the migration path with the smallest $\Delta E_{\mathrm{b}}$

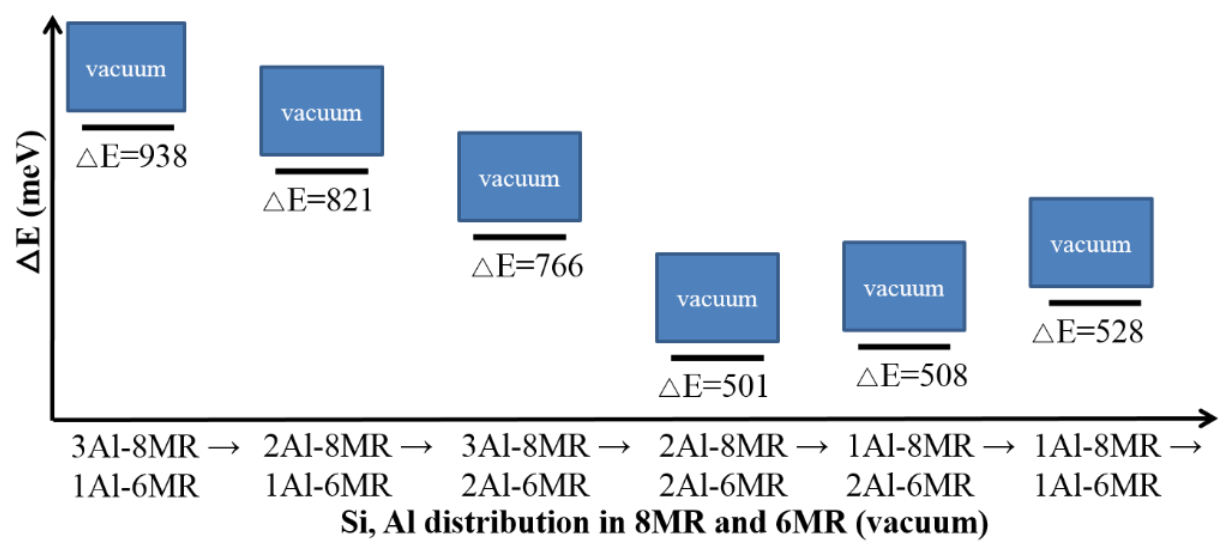

Fig. 5. The influence of uneven $\mathrm{Si}$ and $\mathrm{Al}$ distribution on the energy difference $\Delta E$ for $\mathrm{Cs}^{+}$ occupancy at SIII' and SII in r3CsCHA (in vacuum).

The above discussion is for a bare r3CsCHA system. The presence of gas molecules could change $\Delta E$ (and the $\Delta E_{\mathrm{b}}$ ) results. Figure 6 shows the energy difference $\Delta E$ results of the case where one $\mathrm{CO}_{2}$ molecule was placed near the migration path. The presence of $\mathrm{CO}_{2}$ leads to remarkably reduced $\Delta E$. The relative energy order remains similar to the vacuum case cation migration from Al-richer $8 \mathrm{MRs}$ to Al-leaner 6MRs exhibiting a higher $\Delta E$ value. This consistency suggests that the combination of a 1-Al-contained $8 \mathrm{MR}$ to a 2-Al-contained $6 \mathrm{MR}$ is the migration path for $\mathrm{Cs}^{+}$with the least thermal activation. In next section, we carried out NEB calculations for this case to accurately determine the $\Delta E_{\mathrm{b}}$.

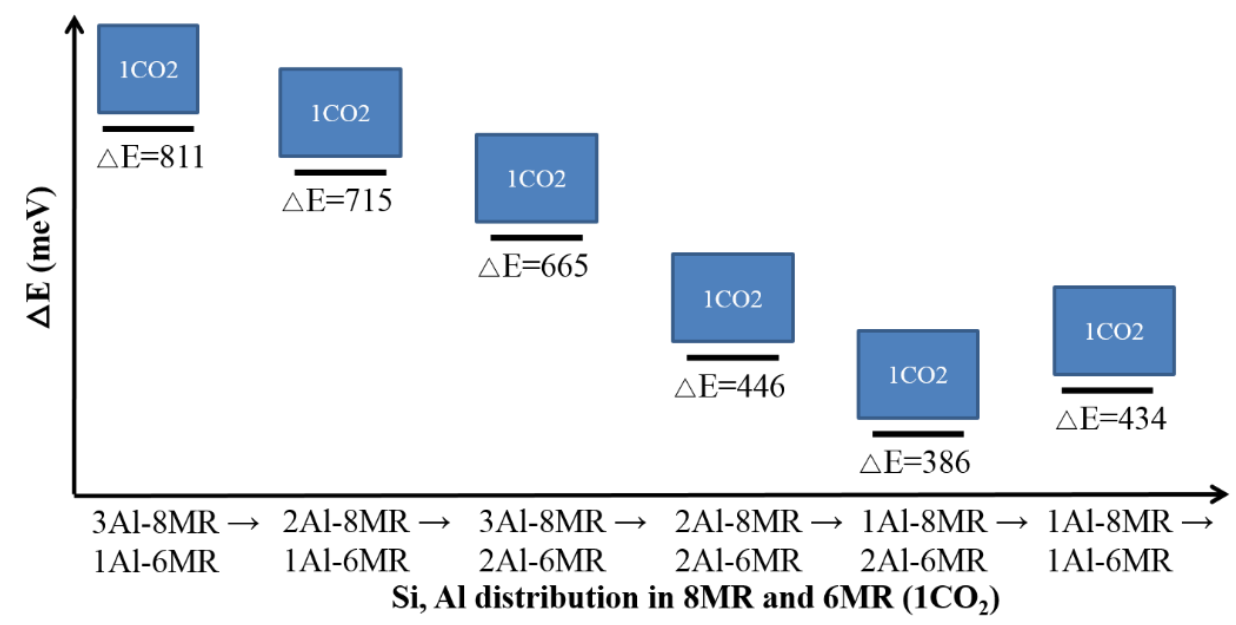


Fig. 6. The influence of uneven $\mathrm{Si}$ and $\mathrm{Al}$ distribution on the energy difference $\Delta E$ for $\mathrm{Cs}^{+}$ occupancy at SIII' and SII in the presence of one $\mathrm{CO}_{2}$ in one $\mathbf{r} 3 \mathrm{CsCHA}$ unit cell.

\subsection{The Effect of Gas Molecules on Cation Movement}

In this section, we consider the influence of $\mathrm{CO}_{2}, \mathrm{~N}_{2}$, and $\mathrm{CH}_{4}$ on the magnitude of the energy barrier $\Delta E_{\mathrm{b}}$ for cation movement along the easiest path. We performed NEB calculations to straightforwardly examine the effect of gas on the energy barrier associated with cation movement path identified in the last section (from the 1-Al contained 8MR (SIII') to the 2-Al contained 6MR (SII)) for selected typical cases. Specifically, we studied the effect of $\mathrm{CO}_{2}$ in detail by considering three different gas concentrations. For $\mathrm{N}_{2}$ and $\mathrm{CH}_{4}$, we only considered two concentrations and one concentration, respectively. The results are shown in Fig. 7. Notably, $\mathrm{CO}_{2}$ significantly reduces the energy barrier for cation migration with respect to vacuum and a higher $\mathrm{CO}_{2}$ concentration leads to larger extent of reduction in the energy barrier. In contrast, both $\mathrm{N}_{2}$ and $\mathrm{CH}_{4}$ impart negligible effect in reducing the energy barrier for cation migration.

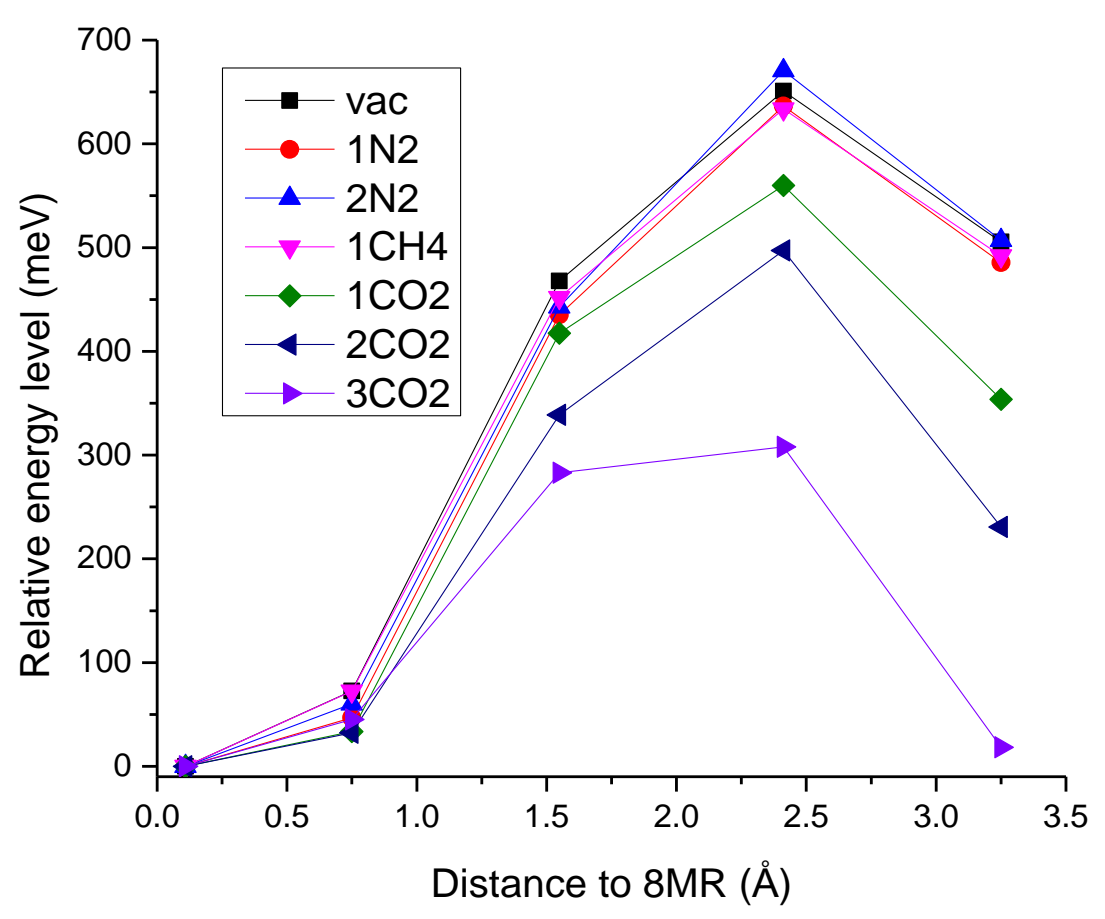

Fig. 7. The effect of different gases on the energy barrier $\Delta E_{\mathrm{b}}$ for one $\mathrm{Cs}^{+}$cation movement from SIII' to SII in r3CsCHA. 


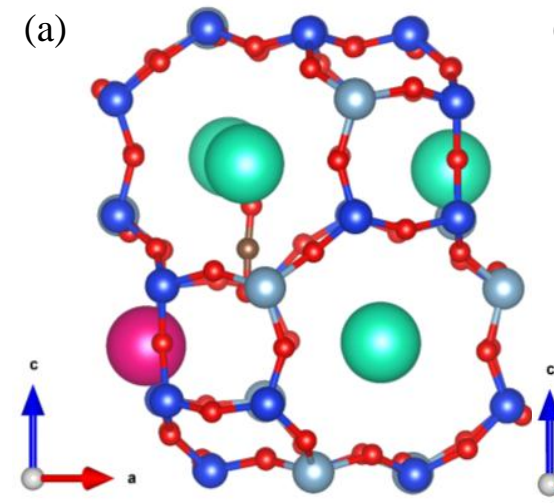

(b)

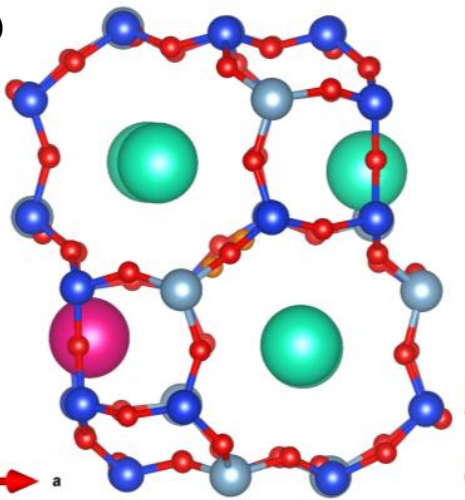

(c)

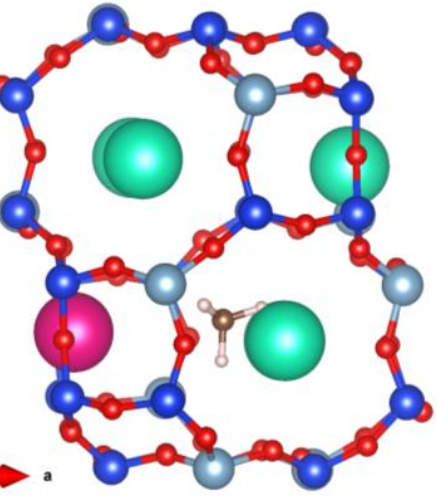

Fig. 8. The gas-adsorbent adsorption complexes in the cases of (a) $\mathrm{CO}_{2}-\mathbf{r} 3 \mathrm{CsCHA}$, (b) $\mathrm{N}_{2}$ r3CsCHA, and (c) $\mathrm{CH}_{4}$-r3CsCHA. Color scheme: Red, O; Blue: Si; Grey, Al; Aqua and violet, Cs; brown: C; Light grey: $\mathrm{N}$ : orange; $\mathrm{H}$ : light pink.

To further justify the viability of using energy difference to represent energy barrier, we also calculated the energy difference results for above cases. For each case, we calculated the total energy of the fully relaxed configuration of gas-chabazite complex with the $\mathrm{Cs}^{+}$cation at the center of 1-Al contained 8MR (SIII') or above the 2-Al contained 6MR (SII), labelled $E_{\text {total }}(\mathrm{SIII})$ and $E_{\text {total }}(\mathrm{SII})$, respectively. The gas concentrations correspond to one, two, or three gas molecules per unit cell. We then simply recorded the absolute difference between the total energy with $\mathrm{Cs}^{+}$at SIII' and that with $\mathrm{Cs}^{+}$at SII as the energy difference, i.e., $\Delta E_{\mathrm{b}}=$ $\left|E_{\text {total }}(\mathrm{SIII} ')-E_{\text {total }}(\mathrm{SII})\right|$. As shown in Table $3, \mathrm{CO}_{2}$ significantly lowers the energy difference with respect to vacuum and increasing the gas concentration strengthens this effect: at $1 \mathrm{CO}_{2}$ per unit cell reduces approximately $25 \% ; 2 \mathrm{CO}_{2}$ approximately $50 \% ; 3 \mathrm{CO}_{2}$ more than $90 \%$. In contrast, neither $\mathrm{N}_{2}$ nor $\mathrm{CH}_{4}$ could effectively lower the energy difference compared with vacuum, even if the gas concentration is elevated. Clearly, the energy difference gives the same trend in the effect of gas molecules on the difficulty for cation migration determined by the energy barrier, consolidating this semi-quantitative method of using $\Delta E$ to represent $\Delta E_{\mathrm{b}}$.

To illustrate the interaction between different gases and chabazite, Figure 8 shows geometry of adsorbate-adsorbent interaction complex of their most stable configurations with $\mathrm{Cs}^{+}$ cation at the $8 \mathrm{MR}$ site (SIII'). The binding energy values (calculated at the optB86b-vdW level) are 51.62, 31.94, $44.42 \mathrm{~kJ} / \mathrm{mol}$ for $\mathrm{CO}_{2}, \mathrm{~N}_{2}$, and $\mathrm{CH}_{4}$ respectively. The adsorbate-Cs distance values are $3.15 \AA$ (between $\mathrm{Cs}^{+}$and the nearest $\mathrm{O}$ of $\mathrm{CO}_{2}$ ), $3.82 \AA$ (between $\mathrm{Cs}^{+}$and 
the nearest $\mathrm{N}$ of $\mathrm{N}_{2}$ ), and $3.69 \AA$ (between $\mathrm{Cs}^{+}$and the nearest $\mathrm{H}$ of $\mathrm{CH}_{4}$ ). It should be noted that the sequence of binding energy of different gases are not directly correlated to the sequence of reduced energy barrier for cation migration. This is because the binding energy reflects the total interaction between the adsorbate and the whole adsorbent (including both the framework and extraframework cation). We contend that there might be a correlation between the Cs-adsorbate interaction and the energy barrier associated to cation migration. However, DFT calculates cannot extract the Cs-adsorbate interaction from the total binding energy. We will attempt to solve this problem in the future work.

Table 3. The effect of different gases on the energy difference (in meV) between SIII' and $\mathrm{SII}$ in $\mathbf{r} 3 \mathrm{CsCHA}$.

\begin{tabular}{|c|c|c|c|}
\hline $\begin{array}{l}\text { Atmosphere } \\
\text { NO. of molecules }\end{array}$ & $\mathrm{CO}_{2}$ & $\mathbf{N}_{2}$ & $\mathrm{CH}_{4}$ \\
\hline 0 & 508 & 508 & 508 \\
\hline 1 & 386 & 500 & 501 \\
\hline 2 & 262 & 501 & 551 \\
\hline 3 & 45 & 450 & 445 \\
\hline
\end{tabular}

Overall, our calculations suggest that $\mathrm{CO}_{2}$ has the strongest ability to reduce the energy barrier for cation movement with respect to under vacuum, while $\mathrm{N}_{2}$ and $\mathrm{CH}_{4}$ have nearly no ability to lower this energy barrier. Increasing the gas concentration magnifies the effect of $\mathrm{CO}_{2}$ but has no influence on $\mathrm{N}_{2}$ or $\mathrm{CH}_{4}$. We attribute the effect of gases on inducing the cation movement to their polarity. $\mathrm{CO}_{2}$ has strong quadrupole and high polarisability, which enables it to strongly interact with the $\mathrm{Cs}^{+}$cation and to flatten the potential energy well of the cation. On the other hand, $\mathrm{N}_{2}$ has smaller quadrupole and polarisability than $\mathrm{CO}_{2}$, and $\mathrm{CH}_{4}$ only has smaller polarisability than $\mathrm{CO}_{2}$, making these two gases interact with the cation too weakly to change its potential energy well.

\subsection{The Effect of Dispersion Correction on the Cation Movement}

Dispersion force plays an indispensable part in the interaction between gas molecules and zeolites [27], as it contributes to the binding energy to some extent. However, it is yet to be known if the contribution from dispersion affects the relative energies between different configurations. We adopted a vdW-DF scheme - optB86b-vdW - to examine its effect on the 
energy difference by selecting the typical cases in $\mathbf{r} 3 \mathrm{CsCHA}$ : vacuum, $1 \mathrm{CH}_{4}$ per unit cell, 1 $\mathrm{N}_{2}$ per unit cell, $1 \mathrm{CO}_{2}$ per unit cell, $2 \mathrm{CO}_{2}$ per unit cell, and $3 \mathrm{CO}_{2}$ per unit cell. The optB86b-vdW scheme represents a typical vdW method as we have determined that it is the most appropriate one in accounting for our gas-chabazite system in a previous study [21]. The results (shown in Fig. 9) clearly show that incorporation of dispersion doses not change the trend of energy difference under any atmosphere; it only lowers the magnitude of the respective energy difference to a similar extent, i.e., $\sim 50 \mathrm{meV}$. This indicates that the dispersion background created by the zeolite framework keeps generally unvaried for the cation and gas molecules at least for the starting ( $\mathrm{Cs}^{+}$at SIII') and ending ( $\mathrm{Cs}^{+}$at SII) configurations of the cation migration path. Therefore, we can safely use results calculated at pure DFT level (PBE level) for evaluation of energy barriers in our system, which effectively cuts the computational cost.

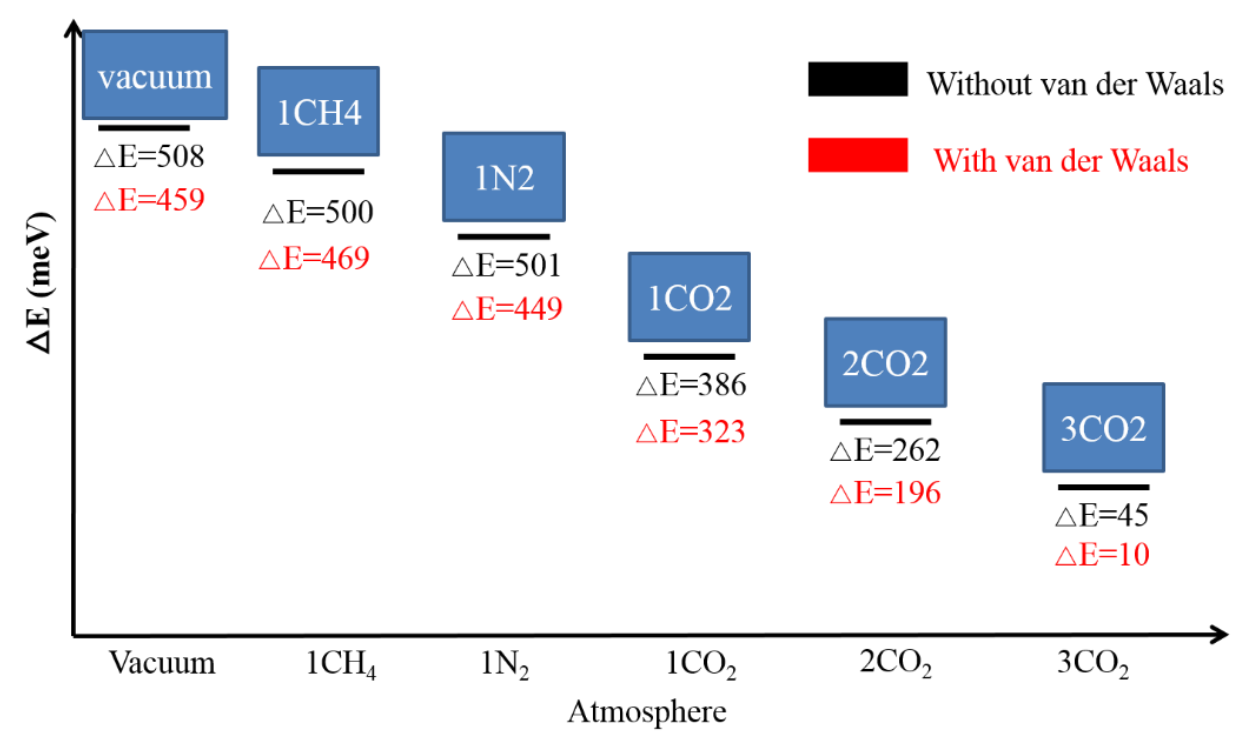

Fig. 9. The effect of vdW dispersion on the energy difference in r3CsCHA.

\subsection{Assessment of viability of trapdoor mechanism to other gas molecules}

In the end of this paper, we would like to utilise the $\Delta E$ (semi-quantitative measure of $\Delta E_{\mathrm{b}}$ ) to examine the viability of trapdoor mechanism on some other gas molecules, such as $\mathrm{H}_{2}, \mathrm{CO}$, $\mathrm{H}_{2} \mathrm{O}, \mathrm{H}_{2} \mathrm{~S}, \mathrm{~N}_{2} \mathrm{O}, \mathrm{C}_{2} \mathrm{H}_{2}$, and $\mathrm{O}_{2}$. 
Table 4 summarises the results. $\mathrm{H}_{2}$ has a weak interaction with $\mathrm{Cs}^{+}$cation owning to its

\begin{tabular}{lllllllll}
\hline Atmosphere & $1 \mathrm{CO}$ & $3 \mathrm{CO}$ & $1 \mathrm{H}_{2}$ & $1 \mathrm{H}_{2} \mathrm{O}$ & $1 \mathrm{H}_{2} \mathrm{~S}$ & $1 \mathrm{~N}_{2} \mathrm{O}$ & $1 \mathrm{C}_{2} \mathrm{H}_{2}$ & $1 \mathrm{O}_{2}$ \\
\hline$\Delta E(\mathrm{meV})$ & 477 & 283 & 498 & 242 & 351 & 356 & 446 & 455 \\
\hline
\end{tabular}

\section{Conclusions}

We have employed density functional theory calculations to demonstrate that the "doorkeeping" $\mathrm{Cs}^{+}$cation has to pay an energy penalty to move away from the center of $8 \mathrm{MR}$ pore aperture (thus allow gas admission) in a typical trapdoor zeolite - r3CsCHA. The difficulty of this "door-open" process can be reflected by the energy barrier associated with the cation movement path from its most stable position (site SIII') to the second most stable one (site SII) inside the chabazite supercavity. The energy difference between these two configurations can qualitatively feature the actual energy barrier. The presence of "strong" gas molecules that possess "strong" interaction strength owning to relatively high polarity, such as $\mathrm{CO}_{2}$ and $\mathrm{CO}$, can substantially lower the energy difference, whereas those "weak" molecules, such as $\mathrm{N}_{2}, \mathrm{CH}_{4}$, and $\mathrm{H}_{2}$, can hardly change the energy difference. The incorporation of van der Waals dispersion does not compromise the above argument. Therefore, we conclude that 
different gases has different abilities to affect the movement of the door-keeping cation in Csexchanged chabazite, reflected by the energy difference between the total energies of the starting and ending configurations. This study lays a concrete theoretical foundation for our proposed "molecular trapdoor" mechanism [5] and directs the theoretical route for further investigation of other novel trapdoor materials.

\section{Acknowledgement}

This research was undertaken with the assistance of resources from the National Computational Infrastructure (NCI), which is supported by the Australian Government. J.S., P.A.W., and J.Z.L acknowledge the Australian Research Council for providing the funding (DP2013000024). G.L. is the recipient of an Australian Research Council Discovery Early Career Researcher Award (DE140101824).

\section{Author contributions}

J.S., P.A.W. and J.Z.L. conceived and designed the molecular simulation study. J.S. and J.Z.L. conducted the $a b$ initio DFT calculations and J.S., G.L., P.A.W., and J.Z.L., analyzed the data. J.S. and G.L. wrote the paper. All authors discussed the results and commented on the manuscript.

Conflict of Interest: The authors declare that they have no conflict of interest.

\section{References}

[1] K. Lee, É.D. Murray, L. Kong, B.I. Lundqvist, D.C. Langreth, Higher-accuracy van der Waals density functional, Physical Review B, 82 (2010) 081101.

[2] J. Shang, G. Li, J. Li, L. Li, P.A. Webley, J.Z. Liu, Density Functional Theory Computational Study of Alkali Cation-Exchanged Sodalite-like Zeolitelike Metal-Organic Framework for CO2, N2, and CH4 Adsorption, The Journal of Physical Chemistry C, 119 (2015) 27449-27456.

[3] T. Ohgushi, K. Ishimaru, Y. Adachi, Movements and Hydration of Potassium Ion in K-A Zeolite, The Journal of Physical Chemistry C, 113 (2009) 2468-2474.

[4] D.W. Breck, Zeolite Molecular Sieves: Structure, Chemistry, and Use, Wiley, New York, 1974.

[5] J. Shang, G. Li, R. Singh, Q. Gu, K.M. Nairn, T.J. Bastow, N. Medhekar, C.M. Doherty, A.J. Hill, J.Z. Liu, P.A. Webley, Discriminative Separation of Gases by a "Molecular Trapdoor" Mechanism in Chabazite Zeolites, J. Am. Chem. Soc., 134 (2012) 19246-19253. 
[6] J. Shang, G. Li, R. Singh, P. Xiao, J.Z. Liu, P.A. Webley, Determination of Composition Range for "Molecular Trapdoor" Effect in Chabazite Zeolite, The Journal of Physical Chemistry C, 117 (2013) 12841-12847.

[7] J. Shang, G. Li, Q. Gu, R. Singh, P. Xiao, J.Z. Liu, P.A. Webley, Temperature controlled invertible selectivity for adsorption of $\mathrm{N} 2$ and $\mathrm{CH} 4$ by molecular trapdoor chabazites, Chem. Commun., 50 (2014) 4544-4546.

[8] O.V. Yakubovich, W. Massa, P.G. Gavrilenko, I.V. Pekov, Crystal structure of chabazite K, Crystallogr. Rep., 50 (2005) 544-553.

[9] J. Shang, G. Li, R. Singh, P. Xiao, J.Z. Liu, P.A. Webley, Potassium Chabazite: A Potential Nanocontainer for Gas Encapsulation, J. Phys. Chem. C., 114 (2010) 22025-22031. [10] W.J. Mortier, J.J. Pluth, J.V. Smith, Positions of cations and molecules in zeolites with the chabazite framework I. Dehydrated ca-exchanged chabazite, Mater. Res. Bull., 12 (1977) 97-102.

[11] W.J. Mortier, J.J. Pluth, J.V. Smith, Positions of cations and molecules in zeolites with the chabazite framework III. Dehydrated Na-exchanged chabazite, Mater. Res. Bull., 12 (1977) 241-249.

[12] M. Calligaris, A. Mezzetti, G. Nardin, L. Randaccio, Cation sites and framework deformations in dehydrated chabazites. Crystal structure of a fully silver-exchanged chabazite, Zeolites, 4 (1984) 323-328.

[13] P. Xiao, J. Zhang, P. Webley, G. Li, R. Singh, R. Todd, Capture of CO2 from flue gas streams with zeolite $13 \mathrm{X}$ by vacuum-pressure swing adsorption, Adsorption, 14 (2008) 575582.

[14] F. Göltl, J. Hafner, Alkane adsorption in Na-exchanged chabazite: The influence of dispersion forces, J. Chem. Phys., 134 (2011) 0641021-06410211.

[15] G. Kresse, J. Furthmüller, Efficient iterative schemes for ab initio total-energy calculations using a plane-wave basis set, Phys. Rev. B, 54 (1996) 11169-11186.

[16] J.P. Perdew, K. Burke, M. Ernzerhof, Generalized Gradient Approximation Made Simple, Phys. Rev. Lett., 77 (1996) 3865-3868.

[17] G. Kresse, D. Joubert, From ultrasoft pseudopotentials to the projector augmented-wave method, Phys. Rev. B, 59 (1999) 1758-1775.

[18] F. Celestini, G. Kirstetter, Effect of an electric field on a Leidenfrost droplet, Soft Matter, 8 (2012) 5992-5995.

[19] P.S. Bárcia, L. Bastin, E.J. Hurtado, J.A.C. Silva, A.E. Rodrigues, B. Chen, Single and Multicomponent Sorption of $\mathrm{CO}_{2}, \mathrm{CH}_{4}$ and $\mathrm{N}_{2}$ in a Microporous Metal-Organic Framework, Sep. Purif. Technol., 43 (2008) 3494-3521.

[20] G.G. Wells, R. Ledesma-Aguilar, G. McHale, K. Sefiane, A sublimation heat engine, Nat Commun, 6 (2015).

[21] J. Shang, G. Li, R. Singh, P. Xiao, D. Danaci, J.Z. Liu, P.A. Webley, Adsorption of CO2, $\mathrm{N} 2$, and $\mathrm{CH} 4$ in Cs-exchanged chabazite: A combination of van der Waals density functional theory calculations and experiment study, The Journal of Chemical Physics, 140 (2014) 084705.

[22] K. Sumida, D.L. Rogow, J.A. Mason, T.M. McDonald, E.D. Bloch, Z.R. Herm, T.-H. Bae, J.R. Long, Carbon Dioxide Capture in Metal-Organic Frameworks, Chem. Rev., 112 (2012) 724-781.

[23] B. Civalleri, A.M. Ferrari, M. Llunell, R. Orlando, M. Mérawa, P. Ugliengo, Cation Selectivity in Alkali-Exchanged Chabazite: An ab Initio Periodic Study, Chem. Mat., 15 (2003) 3996-4004.

[24] M. Calligaris, A. Mezzetti, G. Nardin, L. Randaccio, Crystal structures of the hydrated and dehydrated forms of a partially cesium-exchanged chabazite, Zeolites, 6 (1986) 137-141. 
[25] L.J. Smith, H. Eckert, A.K. Cheetham, Potassium Cation Effects on Site Preferences in the Mixed Cation Zeolite Li,Na-Chabazite, Chem. Mat., 13 (2001) 385-391.

[26] T. Bučko, J. Hafner, The role of spatial constraints and entropy in the adsorption and transformation of hydrocarbons catalyzed by zeolites, J. Catal., 329 (2015) 32-48.

[27] R.E. Morris, J. Cejka, Exploiting chemically selective weakness in solids as a route to new porous materials, Nat Chem, 7 (2015) 381-388. 